\title{
Rejection of Narrow Band Unknown Disturbances in an Active Suspension System
}

\author{
Aurelian Constantinescu, Daniel Rey and Ioan Doré Landau
}

\begin{abstract}
The rejection of unknown disturbances of unknown or varying frequencies is one of the main problems in active vibration control. To achieve the rejection of the disturbances (at least asymptotically) without measuring them, a feedback solution can be considered.

This paper discusses a feedback adaptive control approach for active vibration systems in the presence of unknown narrow band disturbances, with the model of the plant considered known (e.g. obtained by standard system identification). As the controller should incorporate the model of the disturbance, the rejection of unknown disturbances raises the problems of adapting the internal model of the controller and re-designing the controller in real-time.

A direct adaptive control scheme based on the internal model principle and the use of the Youla-Kucera parameterization is proposed. This approach is comparatively evaluated with respect to an indirect adaptive control scheme based on the estimation of the disturbance model. The evaluation of the performance of the adaptive algorithms is illustrated in real time on an active suspension system.
\end{abstract}

\section{INTRODUCTION}

This paper presents the evaluation of two narrow band disturbance attenuation feedback adaptive schemes in the case of an active suspension: a direct and an indirect one.

Several solutions for the attenuation of narrow band disturbances of unknown or varying frequency in active vibration control have been proposed in the literature [1], [2], [3], [4]. These solutions have been inspired by Widrow's technique for adaptive noise cancellation [5] and they require an additional transducer to provide a measurement highly correlated with the unknown disturbance. Two main drawbacks of these approaches can be mentioned: the necessity of an additional transducer (whose location is generally not obvious to determine or to use) and the complexity of the adaptation algorithm due to the important number of adaptation parameters.

Feedback approach is another solution which may be considered for the rejection of the disturbance (at least asymptotically), which does not require any additional measurement. In this case, the common framework is based on the assumption that a narrow band disturbance is the result of a white noise or a Dirac impulse passed through the "model

A. Constantinescu is with the Department of Electrical Engineering, École de technologie supérieure, 1100 Notre-Dame West street, Montréal (QC), H3C1K3, Canada aconstan@ele.etsmt l. ca

D. Rey is with Laboratoire d'Automatique de Grenoble, ENSIEG, BP 46, 38402 Saint Martin d'Hères, France daniel.reyeinpg. fr

I.D. Landau is with Laboratoire d'Automatique de Grenoble, ENSIEG, BP 46, 38402 Saint Martin d'Hères, France landau.ioan@aliceadsl.fr of the disturbance"1.

The solution presented in this paper for the case of the active suspension system considers that the plant model is known and the disturbance model is unknown ${ }^{2}$; the model of the plant can be obtained by standard system identification and does not normally change during operation. One of the approaches considered for solving this problem is the use of the internal model principle [9], [10], [11], [12], [13], [14], [15], [16], but other approaches may be also considered, such as the use of an observer for the disturbance [17], [18] or the use of a "phase-locked" loop structure [19].

Since the parameters of the disturbance model are unknown all these approaches lead to an adaptive implementation which can be of direct or indirect type. These two implementation approaches for the rejection of time-varying unknown narrow band disturbances, presented and evaluated in this paper, are based on the internal model principle. As such, the controller should incorporate the model of the disturbance [9], [10], [11], [12]. Hence, the rejection of unknown disturbances becomes a problem of adapting the internal model of the controller and of re-designing it in real-time.

In the indirect adaptive control scheme, the model of the disturbance is estimated and the controller is re-computed in real time; in this case the controller should incorporate the estimated model of the disturbance as a pre-specified element of the controller.

The other solution would be to consider the Youla-Kucera parametrization of the controller (known also as the Qparametrization). In this case it is possible to insert and adjust the internal model in the controller by adjusting the parameters of a polynomial $Q$ (see Fig. 1). This approach leads to a direct adaptive control scheme where the parameters of the polynomial $Q$ are directly adapted in order to have the desired internal model; the polynomials $R_{0}$ and $S_{0}$ do not change, so there is no need to recompute the controller in real-time. The computational volume of the adaptation algorithm depends upon the complexity of the disturbance model.

From the user point of view and taking into account the type of operation of existing adaptive disturbance compensation systems, two modes of operation of the adaptive schemes

\footnotetext{
${ }^{1}$ Throughout the paper it is assumed that the order of the disturbance model is known but the parameters of the model are unknown.

${ }^{2}$ Other cases may be also considered, such as unknown plant and disturbance model or unknown plant and known disturbance model, which have been considered in the literature (see for example [6] and [7], [8], respectively).
} 


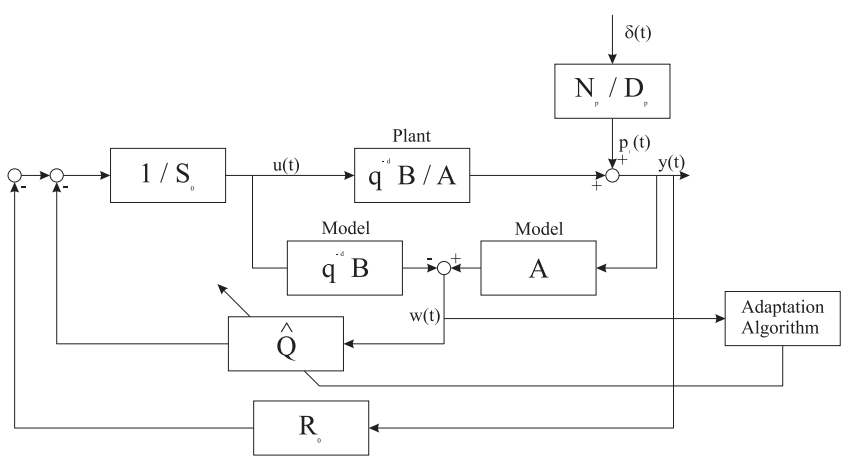

Fig. 1. Direct adaptive control scheme for rejection of unknown disturbances

may be considered:

1) Self-tuning operation: the adaptation procedure starts either on demand or when the performance is unsatisfactory (automatically). In this situation the current controller is frozen during the computation of the new controller parameters.

2) Adaptive operation: the adaptation is performed continuously and the controller parameters are updated at each sampling.

This paper focuses on the results obtained with a direct feedback adaptive control algorithm for the case of unknown and time-varying frequency narrow band disturbances applied to an active suspension. The direct adaptive control scheme uses the Youla-Kucera parametrization for the computation of the controller. This algorithm takes its roots from an idea of Tsypkin [22].

For evaluation purposes (complexity and performance) an indirect adaptive control scheme based on the Internal Model Principle is also presented and the results are evaluated comparatively.

The paper is organized as follows. In section II the active suspension system on which the algorithms have been tested is presented. Section III is dedicated to a brief review of the plant, disturbance and controller representation as well as of the Q-parametrization. The direct adaptive control scheme is presented in section IV and the indirect one in section V. Section VI presents the results obtained in real time on the active suspension. Some concluding remarks are given in section VII.

\section{ThE ACTIVE SUSPENSION SYSTEM}

The structure of the system (the active suspension) used in this paper is presented in Fig. 2. It consists of the active suspension, a load, a shaker and the components of the control scheme. The mechanical construction of the load is such that the vibrations produced by the shaker, fixed to the ground, are transmitted to the upper side of the active suspension. The active suspension is based on a hydraulic system allowing to reduce the over-pressure at the frequencies of the vibration modes of the suspension. Its components are: an elastomer cone (1) which marks the main chamber filled up with silicon oil, a secondary chamber (2)

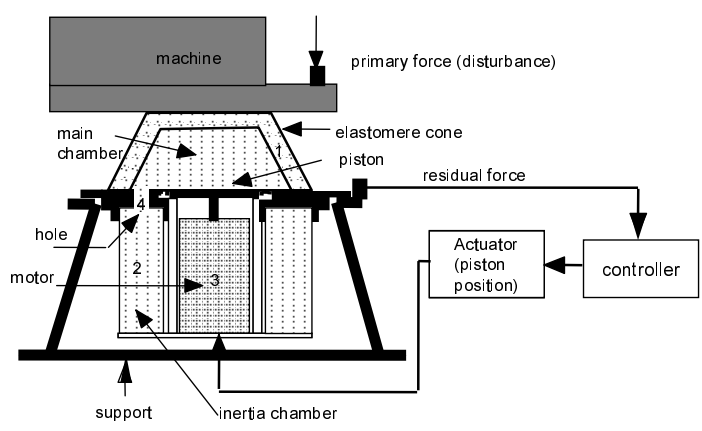

Fig. 2. Active suspension system (scheme)

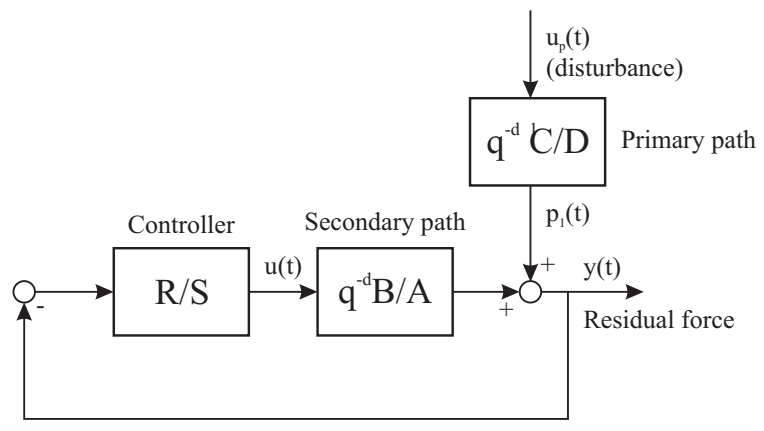

Fig. 3. Block diagram of the active suspension system

marked by a flexible membrane, a piston (3) attached to a motor, an orifice (4) allowing the oil to pass between the two chambers and a force sensor located between the support and the active suspension.

The controller will act upon the piston (through a power amplifier) in order to reduce the residual force. The sampling frequency is $800 \mathrm{~Hz}$. The equivalent control scheme is shown in Fig. 3. The system input, $u(t)$ is the position of the piston (see Fig. 2 and Fig. 3), the output $y(t)$ is the residual force measured by a force sensor. The transfer function $\left(q^{-d_{1}} C / D\right)$, between the disturbance force, $u_{p}$, and the residual force $y(t)$ is called primary path. In our case (for testing purposes), the primary force is generated by a shaker controlled by a signal given by the computer. The transfer function $\left(q^{-d} B / A\right)$ between the input of the system, $u(t)$, and the residual force is called secondary path. The input of the system being a position and the output a force, the secondary path transfer function has a double differentiator behavior.

The control objective is to reject the effect of unknown narrow band disturbances on the output of the system (residual force) - i.e. attenuate the vibrations transmitted from the machine to the support via the active suspension. The physical parameters of the active suspension system are not provided by the manufacturer. The system has to be considered as a "black box". 


\section{BASIC NOTATIONS AND PROBLEM FORMULATION}

\section{A. Plant representation and controller structure}

The structure of a linear time invariant discrete time model of the plant (used for controller design) is:

$$
G\left(z^{-1}\right)=\frac{z^{-d} B\left(z^{-1}\right)}{A\left(z^{-1}\right)}=\frac{z^{-d-1} B^{*}\left(z^{-1}\right)}{A\left(z^{-1}\right)},
$$

where:

$$
\begin{aligned}
d & =\text { plant pure time delay [no. of sampling periods]; } \\
A & =1+a_{1} z^{-1}+\ldots+a_{n_{A}} z^{-n_{A}} \\
B & =b_{1} z^{-1}+\ldots+b_{n_{B}} z^{-n_{B}}=q^{-1} B^{*}
\end{aligned}
$$

$A\left(z^{-1}\right), B\left(z^{-1}\right), B^{*}\left(z^{-1}\right)$ are polynomials in the complex variable $z^{-1}$ and $n_{A}, n_{B}$ and $n_{B}-1$ represent their orders ${ }^{3}$. The model of the plant is obtained by system identification of the secondary path (for details on the system identification of the model of the active suspension see [24], [25], [26], [27], [28]).

The controller to be designed is a RS-type polynomial [29], [30] controller (see Fig. 3). The output of the plant $y(t)$ and the input $u(t)$ may be written as:

$$
\begin{aligned}
y(t) & =\frac{q^{-d} B\left(q^{-1}\right)}{A\left(q^{-1}\right)} \cdot u(t)+p_{1}(t) ; \\
S\left(q^{-1}\right) \cdot u(t) & =-R\left(q^{-1}\right) \cdot y(t),
\end{aligned}
$$

where $q^{-1}$ is the delay (shift) operator ${ }^{4}, p_{1}(t)$ is the resulting additive disturbance on the output of the system. $R\left(z^{-1}\right)$, $S\left(z^{-1}\right)$ are polynomials in $z^{-1}$ having the orders $n_{R}, n_{S}$, with the following expressions :

$$
\begin{aligned}
R\left(z^{-1}\right) & =r_{0}+r_{1} z^{-1}+\ldots+r_{n_{R}} z^{-n_{R}} \\
& =R^{\prime}\left(z^{-1}\right) \cdot H_{R}\left(z^{-1}\right) \\
S\left(z^{-1}\right) & =1+s_{1} z^{-1}+\ldots+s_{n_{S}} z^{-n_{S}} \\
& =S^{\prime}\left(z^{-1}\right) \cdot H_{S}\left(z^{-1}\right)
\end{aligned}
$$

where $H_{R}$ and $H_{S}$ are pre-specified parts of the controller (used for example to incorporate the internal model of a disturbance or to open the loop at certain frequencies).

Using (1) and (2), the output of the system can be written as

$$
y(t)=\frac{A\left(q^{-1}\right) S\left(q^{-1}\right)}{P\left(q^{-1}\right)} \cdot p_{1}(t)=S_{y p}\left(q^{-1}\right) \cdot p_{1}(t),
$$

where

$$
P\left(z^{-1}\right)=A\left(z^{-1}\right) S\left(z^{-1}\right)+z^{-d} B\left(z^{-1}\right) R\left(z^{-1}\right)
$$

defines the poles of the closed loop and $S_{y p}$ is the output sensitivity function (the transfer function between the disturbance $p_{1}(t)$ and the output of the system $\left.y(t)\right)$.

For more details on RS-type controllers and sensitivity functions see [29].

\footnotetext{
${ }^{3}$ The complex variable $z^{-1}$ will be used for characterizing the system behavior in the frequency domain and the delay operator $q^{-1}$ will be used for describing the system behavior in the time domain.

${ }^{4} x(t)=q^{-1} x(t+1)$
}

\section{B. Problem formulation}

Suppose that $p_{1}(t)$ is a deterministic disturbance, so it can be written as

$$
p_{1}(t)=\frac{N_{p}\left(q^{-1}\right)}{D_{p}\left(q^{-1}\right)} \cdot \delta(t),
$$

where $\delta(t)$ is a Dirac impulse and $N_{p}\left(z^{-1}\right)$ and $D_{p}\left(z^{-1}\right)$ are coprime polynomials in $z^{-1}$, of degrees $n_{N_{p}}, n_{D_{p}}$. We are interested in the rejection of narrow band disturbances and in this case the roots of $D_{p}\left(z^{-1}\right)$ are on the unit circle ${ }^{5}$. The energy of the disturbance is essentially represented by $D_{p}$. The contribution of the terms of $N_{p}$ is weak compared to the effect of $D_{p}$, so one can neglect the effect of $N_{p}$.

In order to apply the Internal Model Principle (to introduce the disturbance model into the controller) we shall consider the pre-specified part of $S\left(z^{-1}\right)$ as $H_{S}\left(z^{-1}\right)=D_{p}\left(z^{-1}\right)$. For details on the Internal Model Principle see [9]. Hence the controller is computed using (6), where $P, D_{p}, A, B$, $H_{R}$ and $d$ are given ${ }^{6}$.

\section{Q-parametrization}

The Youla-Kucera parametrization ( $Q$-parametrization) for disturbance rejection has been explicitely proposed by $\mathrm{Y}$. Z. Tsypkin in [12]. Let $\left[R_{0}\left(z^{-1}\right), S_{0}\left(z^{-1}\right)\right]$ be the nominal controller (without internal model of the disturbance), verifying the diophantine equation (6) and satisfying the imposed robustness constraints. The control law is

$$
S_{0}\left(q^{-1}\right) \cdot u(t)=-R_{0}\left(q^{-1}\right) \cdot y(t) .
$$

Using the Q-parametrization of all stable controllers [23], [12], the controller polynomials $R\left(z^{-1}\right)$ and $S\left(z^{-1}\right)$ get the form:

$$
\begin{aligned}
R\left(z^{-1}\right) & =R_{0}\left(z^{-1}\right)+A\left(z^{-1}\right) Q\left(z^{-1}\right) \\
S\left(z^{-1}\right) & =S_{0}\left(z^{-1}\right)-z^{-d} B\left(z^{-1}\right) Q\left(z^{-1}\right) .
\end{aligned}
$$

The central controller $\left(R_{0}, S_{0}\right)$ can be computed by poles placement (but any other design technique can be used). Given the plant model $(A, B, d)$ and the desired closed-loop poles $P$ one has to solve:

$$
P\left(z^{-1}\right)=A\left(z^{-1}\right) S_{0}\left(z^{-1}\right)+z^{-d} B\left(z^{-1}\right) R_{0}\left(z^{-1}\right) .
$$

Equations (8) and (9) characterize the set of all stabilizable controllers assigning the closed loop poles as defined by $P\left(z^{-1}\right)$. For the purpose of this paper $Q\left(z^{-1}\right)$ is considered to be a polynomial of the form:

$$
Q\left(z^{-1}\right)=q_{0}+q_{1} z^{-1}+\ldots+q_{n_{Q}} z^{-n_{Q}} .
$$

To compute $Q\left(z^{-1}\right)$ in order that the controller incorporates the internal model of the disturbance one has to solve the diophantine equation:

$$
S^{\prime}\left(z^{-1}\right) D_{p}\left(z^{-1}\right)+z^{-d} B\left(z^{-1}\right) Q\left(z^{-1}\right)=S_{0}\left(z^{-1}\right),
$$

\footnotetext{
${ }^{5}$ Since the external disturbance is narrow band, the filtering effect of the primary path around the central frequency can be approximated by a gain and phase lag which will be captured by $N_{p}$.

${ }^{6}$ It is assumed that $D_{p}$ and $B$ do not have common factors.
} 
where $D_{p}\left(z^{-1}\right), d, B\left(z^{-1}\right)$ and $S_{0}\left(z^{-1}\right)$ are known and $S^{\prime}\left(z^{-1}\right)$ and $Q\left(z^{-1}\right)$ are unknown. Equation (12) has a unique solution for $S^{\prime}\left(z^{-1}\right)$ et $Q\left(z^{-1}\right)$ with: $n_{S_{0}} \leq n_{D_{p}}+$ $n_{B}+d-1, n_{S^{\prime}}=n_{B}+d-1, n_{Q}=n_{D_{p}}-1$. It may be seen that the order $n_{Q}$ of the polynomial $\mathrm{Q}$ depends upon the structure of the disturbance model.

\section{DIRECT ADAPTIVE CONTROL}

\section{A. Known Parameters Case}

In the case when the parameters of $D_{p}\left(z^{-1}\right)$ are known, we compute $Q\left(z^{-1}\right)$ by solving the diophantine equation (12), the controller being obtained using the relations (8) and (9).

\section{B. Unknown Parameters Case}

The objective is to find an estimation algorithm which will directly estimate the parameters of the internal model in the controller in the presence of an unknown disturbance (but of known structure) without modifying the closed loop poles. The Q-parametrization is a potential option since modifications of the $Q$ polynomial will not affect the closed loop poles. In order to build an estimation algorithm it is necessary to define an error equation which will reflect the difference between the optimal $Q$ polynomial and its current value.

In [12], such an error equation is provided and it can be used for developing a direct adaptive control scheme. Using the Q-parametrization, the output of the system in the presence of a disturbance can be expressed as:

$$
\begin{aligned}
y(t) & =\frac{A\left(q^{-1}\right)\left[S_{0}\left(q^{-1}\right)-q^{-d} B\left(q^{-1}\right) Q\left(q^{-1}\right)\right]}{P\left(q^{-1}\right)} \\
& \cdot \frac{N_{p}\left(q^{-1}\right)}{D_{p}\left(q^{-1}\right)} \cdot \delta(t) \\
& =\frac{S_{0}\left(q^{-1}\right)-q^{-d} B\left(q^{-1}\right) Q\left(q^{-1}\right)}{P\left(q^{-1}\right)} \cdot w(t),
\end{aligned}
$$

where $\mathrm{w}(\mathrm{t})$ is given by (see also Fig. 1):

$w(t)=\frac{A\left(q^{-1}\right) N_{p}\left(q^{-1}\right)}{D_{p}\left(q^{-1}\right)} \cdot \delta(t)=A\left(q^{-1}\right) \cdot y(t)-q^{-d} \cdot B\left(q^{-1}\right) \cdot u(t)$

In the time domain, the internal model principle can be interpreted as finding $Q$ such that asymptotically $y(t)$ becomes zero.

Consider the estimated polynomial $\hat{Q}\left(t, q^{-1}\right)$ as $\hat{Q}\left(t, q^{-1}\right)=\hat{q}_{0}(t)+\hat{q}_{1}(t) q^{-1}+\ldots+\hat{q}_{n_{Q}}(t) q^{-n_{Q}}$, the associated estimated parameter vector $\hat{\theta}(t)=$ $\left[\hat{q}_{0}(t) \hat{q}_{1}(t) \ldots \hat{q}_{n_{Q}}(t)\right]^{T}$ and the observation vector $\phi^{T}(t)=\left[\begin{array}{ll}w_{2}(t) & w_{2}(t-1) \ldots w_{2}\left(t-n_{Q}\right)\end{array}\right]$, where

$$
w_{2}(t)=\frac{q^{-d} B^{*}\left(q^{-1}\right)}{P\left(q^{-1}\right)} \cdot w(t) .
$$

After computations, the a priori and a posteriori adaptation errors may be written as (for details see [31]):

$$
\begin{aligned}
\varepsilon^{0}(t+1) & =w_{1}(t+1)-\hat{\theta}^{T}(t) \phi(t) \\
\varepsilon(t+1) & =w_{1}(t+1)-\hat{\theta}^{T}(t+1) \phi(t),
\end{aligned}
$$

with

$$
\begin{aligned}
w_{1}(t+1) & =\frac{S_{0}\left(q^{-1}\right)}{P\left(q^{-1}\right)} \cdot w(t+1) \\
w(t+1) & =A\left(q^{-1}\right) y(t+1)-q^{-d} B^{*}\left(q^{-1}\right) u(t)
\end{aligned}
$$

For the estimation of the parameters of $\hat{Q}\left(t, q^{-1}\right)$ the following parameter adaptation algorithm is used:

$$
\begin{aligned}
\hat{\theta}(t+1) & =\hat{\theta}(t)+F(t) \phi(t) \varepsilon(t+1) ; \\
\varepsilon(t+1) & =\frac{\varepsilon^{0}(t+1)}{1+\phi^{T}(t) F(t) \phi(t)} ; \\
\varepsilon^{0}(t+1) & =w_{1}(t+1)-\hat{\theta}^{T}(t) \phi(t) ; \\
F(t+1) & =\frac{1}{\lambda_{1}(t)}\left[F(t)-\frac{F(t) \phi(t) \phi^{T}(t) F(t)}{\frac{\lambda_{1}(t)}{\lambda_{2}(t)}+\phi^{T}(t) F(t) \phi(t)}\right]
\end{aligned}
$$

where $\lambda_{1}(t)$ and $\lambda_{2}(t)$ allow to adjust the adaptation speed (for details see [29]).

In order to implement this methodology for disturbance rejection (see Fig. 1), it is supposed that the plant model $\frac{z^{-d} B\left(z^{-1}\right)}{A\left(z^{-1}\right)}$ is known (identified) and that it exists a controller $\left[R_{0}\left(z^{-1}\right), S_{0}\left(z^{-1}\right)\right]$ which verifies the desired specifications in the absence of the disturbance. One also supposes that the degree $n_{Q}$ of the polynomial $Q\left(z^{-1}\right)$ is fixed, $n_{Q}=n_{D_{p}}-1$ (i.e. the structure of the disturbance is known).

The following procedure is applied at each sampling time for adaptive operation:

1) Get the measured output $y(t+1)$ and the applied control $u(t)$ to compute $w(t+1)$ using (17).

2) Compute $w_{1}(t+1)$ and $w_{2}(t)$ using (16) and (15) with $P$ given by (10).

3) Estimate the $Q$-polynomial using the parametric adaptation algorithm (18) - (21).

4) Compute and apply the control (see Fig. 1):

$$
S_{0}\left(q^{-1}\right) \cdot u(t+1)=-R_{0}\left(q^{-1}\right) \cdot y(t+1)-\hat{Q}\left(t, q^{-1}\right) \cdot w(t+1) .
$$

$t$, For the self tuning operation of the adaptive scheme, the estimation of the $Q$ - polynomial starts once the level of the output is over a defined threshold. A parameter adaptation algorithm (18) - (21) with asymptotically decreasing adaption gain is used and the estimation is stopped when the adaptation gain is below a pre-specified level. During the estimation of new parameters, the controller is kept constant. The controller is updated once the estimation phase is finished.

An analysis of the stability of the algorithm may be found in [31].

\section{INDIRECT ADAPTIVE CONTROL}

The indirect adaptive control methodology for the attenuation of narrow band disturbances consists in two steps:

1) identification of the disturbance model;

2) computation of a digital controller using the identified disturbance model. 
The disturbance is considered as a stationary signal having a rational spectrum. As such it may be considered as the output of a filter with the transfer function $N_{p}\left(z^{-1}\right) / D_{p}\left(z^{-1}\right)$ and a white noise as input:

$$
y(t)=\frac{N_{p}\left(q^{-1}\right)}{D_{p}\left(q^{-1}\right)} \cdot e(t),
$$

where $e(t)$ represents a gaussian white noise, and

$$
\begin{aligned}
& N_{p}\left(z^{-1}\right)=1+n_{p_{1}} z^{-1}+\ldots+n_{p_{n_{N}}} z^{-n_{N_{p}}} \\
& D_{p}\left(z^{-1}\right)=1+d_{p_{1}} z^{-1}+\ldots+d_{p_{n_{D}}} z^{-n_{D_{p}}} .
\end{aligned}
$$

Therefore the disturbance model can be represented by an ARMA model. As we deal with narrow band disturbances, the filtering effect of the primary path in cascade with the output sensitivity function (when operating in closed loop) around the central frequency of the disturbance can be approximated by a gain and a phase lag which will be captured by the $N_{p}\left(z^{-1}\right) / D_{p}\left(z^{-1}\right)$ model.

From (23) one obtains:

$y(t+1)=-\sum_{i=1}^{n_{D_{p}}} d_{p_{i}} y(t-i+1)+\sum_{i=1}^{n_{N_{p}}} n_{p_{i}} e(t-i+1)+e(t+1)$.

The problem is, in fact, an on-line adaptive estimation of parameters in presence of noise [29], [20]. Equation (24) is a particular case of identification of an ARMAX model. The Recursive Extended Least Squares method [29] may be used, for example, which is dedicated to the identification of this type of model. The parameter adaptation algorithm given in (18) - (21) is used. The controller parameters are frozen while the disturbance model is identified. Once the disturbance model is identified, the controller containing the disturbance dynamics is computed by solving the diophantine equation (6) and using (4) with $H_{S}\left(z^{-1}\right)=\hat{D}_{p}\left(z^{-1}\right)$ (the identified model of the disturbance). In order to apply this methodology we suppose that the plant model is known (e.g. obtained by identification). We also suppose that the degrees $n_{N_{p}}$ and $n_{D_{p}}$ of $N_{p}\left(z^{-1}\right)$ respectively $D_{p}\left(z^{-1}\right)$ are fixed.

\section{RESULTS OBTAINED ON THE ACTIVE SUSPENSION}

The narrow band disturbance rejection procedure using the direct and indirect adaptive control schemes proposed in sections IV and V, is illustrated in real time for the case of the control of an active suspension (presented in section II). In our case the disturbance will be a time-varying frequency sinusoid, so we shall consider $n_{D_{p}}=2$ and $n_{Q}=n_{D_{p}}-1=$ 1.

The identification procedure in open and closed-loop operation for the active suspension is discussed in detail in [24], [25], [26], [27], [28]. The frequency characteristic of the identified primary path model (open-loop identification), between the signal $u_{p}$ sent to the shaker in order to generate the disturbance and the residual force $y(t)$, is presented in Fig. 4. The first vibration mode of the primary path model is near $32 \mathrm{~Hz}$. The frequency characteristic of the identified secondary path model (closed-loop identification),

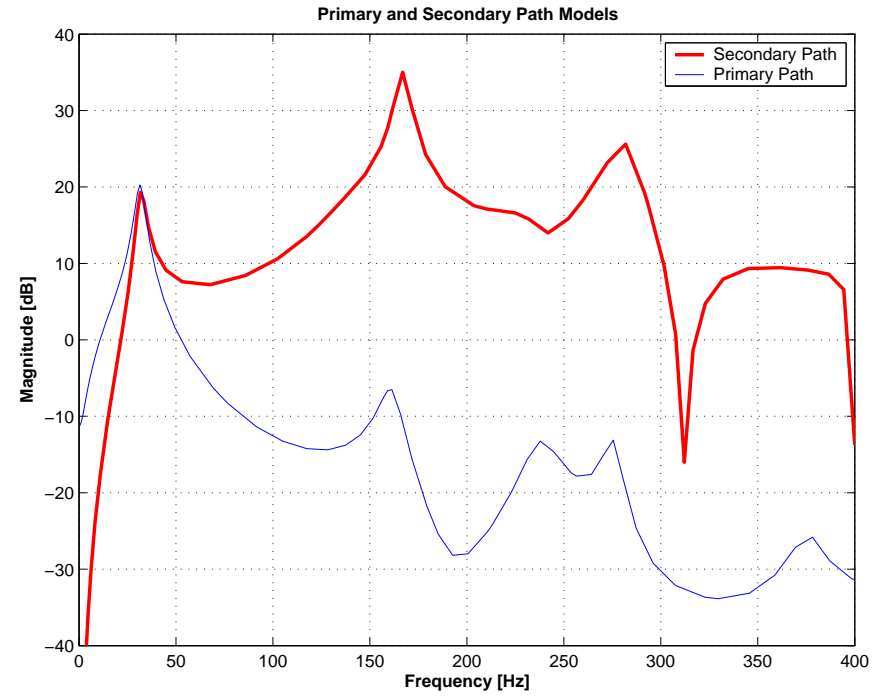

Fig. 4. Frequency characteristics of the primary and secondary path

is presented also in Fig. 4. This model has the following complexity: $n_{B}=14, n_{A}=16, d=0$. The identification has been done using as excitation of the piston a PRBS (Pseudo Random Binary Sequence) with frequency divider $p=4$ (for details on the PRBS signals see [29]). There exist several low damped vibration modes on the secondary path, the first one being at $31.8 \mathrm{~Hz}$ with a damping factor 0.07 . The identified model of the secondary path has been used for the design and implementation of the controller.

The central controller (without the internal model of the disturbance) has been designed using the pole placement method and the secondary path identified model. A pair of dominant poles has been fixed at the frequency of the first vibration mode $(31.8 \mathrm{~Hz})$, with a damping $\xi=0.8$, and the other poles of the model have been considered as auxiliary desired closed loop poles. In addition a pre-specified part $H_{R}=1+q^{-1}\left(R=H_{R} R^{\prime}\right)$ which assures the opening of the loop at $0.5 f_{s}$ has been introduced and 10 auxiliary poles at 0.7 have been added to the desired closed-loop poles. The resulting nominal controller has the following complexity: $n_{R}=14, n_{S}=16$ and it satisfies the imposed robustness constraints on the sensitivity functions ${ }^{7}$.

\section{A. Frequency hopping sinusoidal disturbances (direct and indirect adaptive control)}

In order to evaluate the performance of the direct and indirect methods in real time, frequency hopping sinusoidal disturbances between 25 and $47 \mathrm{~Hz}$ have been used (the first vibration mode of the primary path is near $32 \mathrm{~Hz}$ ).

For both direct and indirect adaptive control methods, two protocols have been defined: one for a self-tuning operation, the other for an adaptive operation.

- Protocol 1 : Self-tuning operation The system operates in closed loop with a frozen controller. As soon as a change of the disturbance is

\footnotetext{
${ }^{7}$ Any design method allowing to satisfy these constraints can be used.
} 


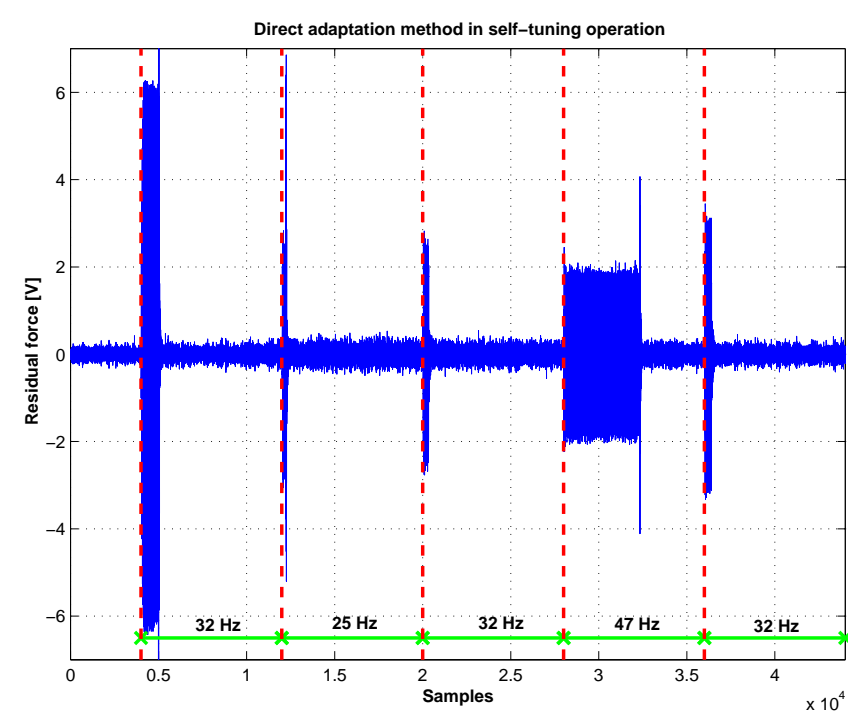

Fig. 5. Time domain results with the direct adaptation method in self-tuning operation

detected (by measuring the variance of the residual output), the estimation algorithm is started with the last frozen controller in operation. When the algorithm converges (a criterion has to be defined), a new controller is computed and applied to the system. The adaptation algorithm is stopped and one waits for a change of frequency.

\section{- Protocol 2 : Adaptive operation}

The estimation algorithm works continuously (once the loop is closed) and the controller is recomputed at each sampling. The adaptation gain in this case does not tend asymptotically to zero.

- Start up: For comparison purposes the system is started in open-loop for both protocols. After 5 seconds (4000 samples) a sinusoidal disturbance of $32 \mathrm{~Hz}$ is applied on the shaker. The model of the disturbance is estimated and an initial controller is computed (same initial controller for both direct and indirect adaptive control). In the case of the self-tuning operation the adaptation algorithm is stopped while in the case of the adaptive operation the adaptation algorithm continues to be active.

After the start up ends, every 10 seconds (8000 samples) sinusoidal disturbances of different frequency are applied $(32 \mathrm{~Hz}, 25 \mathrm{~Hz}, 32 \mathrm{~Hz}, 47 \mathrm{~Hz}, 32 \mathrm{~Hz})$.

1) Protocol 1 : Self-tuning operation. Real time experimental results: The measured residual forces obtained in self-tuning operation with the direct and indirect adaptation methods are presented in Fig. 5 and Fig. 6, respectively. We note in general a faster convergence speed of the direct adaptive control scheme compared to the indirect one (except for $47 \mathrm{~Hz})$.

For the self-tuning protocol, the spectral densities of the residual force obtained in open loop respectively in closed loop using the direct adaptation scheme (after the algorithm converges) are presented in Fig. 7, for the three frequencies

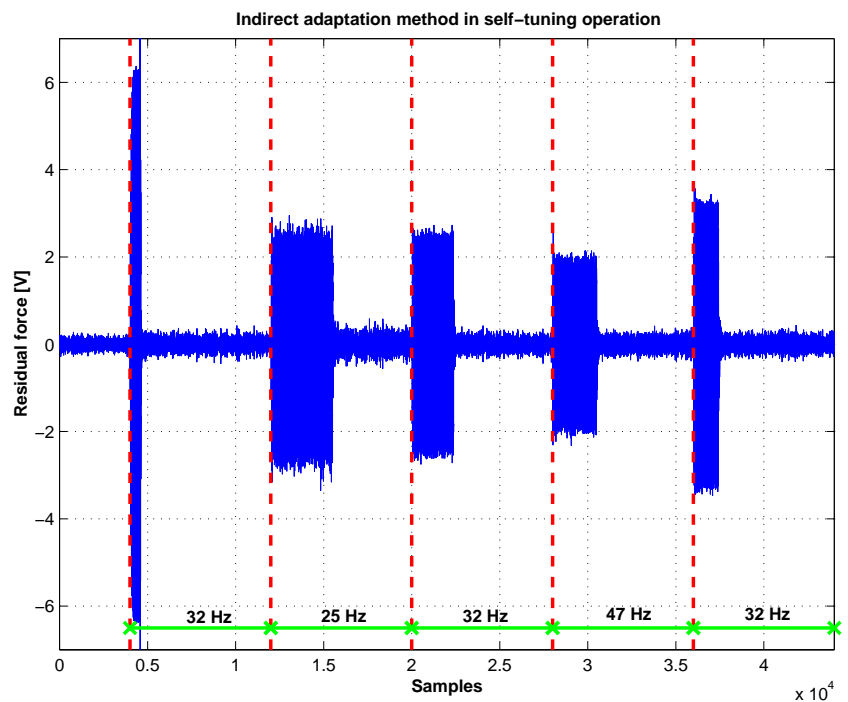

Fig. 6. Time domain results with the indirect adaptation method in selftuning operation

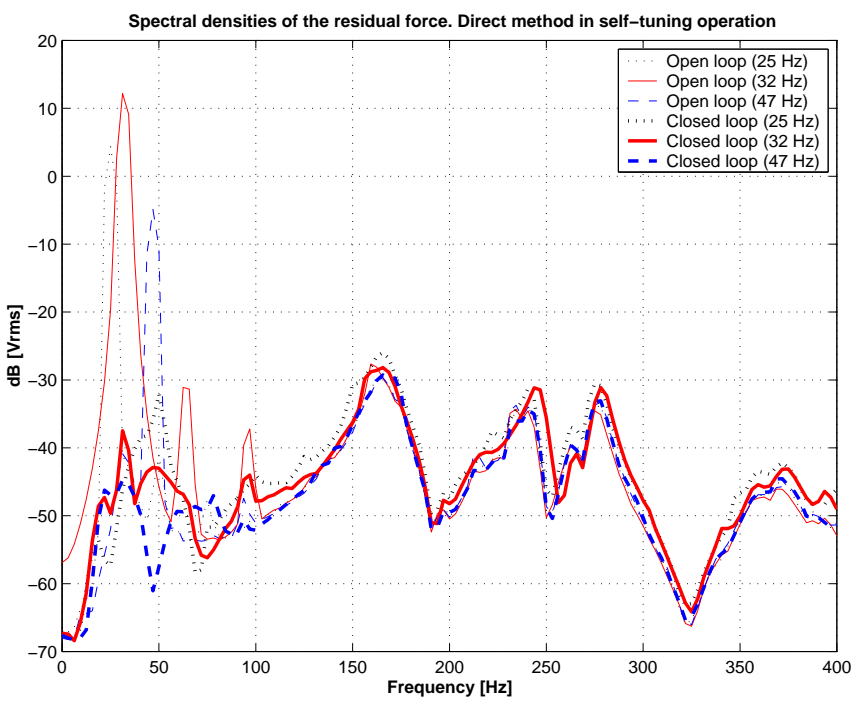

Fig. 7. Spectral densities of the residual force in open and in closed loop, with the direct adaptation method in self-tuning operation

used: 25,32 and $47 \mathrm{~Hz}$. We remark that the attenuations are larger than $49 \mathrm{~dB}$ for all frequencies considered. Similar results are obtained with the indirect adaptation (for details see [24]).

We note the appearance of two harmonics of the first vibration mode of the primary path on the spectral density in open loop when the frequency of the disturbance corresponds with the first resonance mode of the system $(32 \mathrm{~Hz})$. They appear in open loop because of the non-linearities of the system at large signals (there is an important amplification of the disturbance at the resonance frequency of the system in open loop). The harmonics do not appear in closed loop operation.

In self-tuning operation, one uses an adaptation gain $F(t)$ with variable forgetting factor, with $\lambda_{0}=0.97$ and the initial 


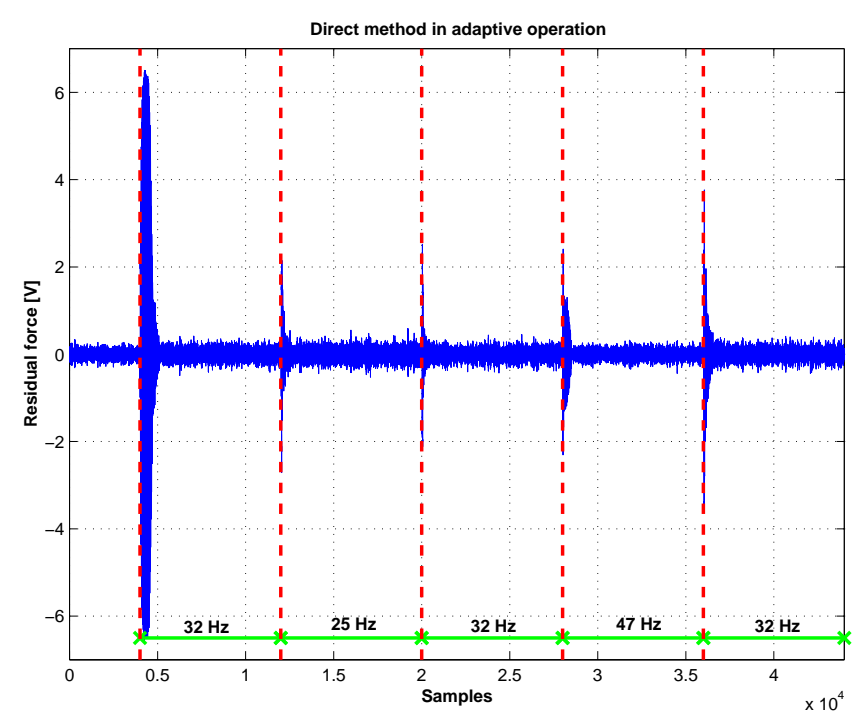

Fig. 8. Time domain results with the direct adaptation method in the adaptive case (trace $=3 \cdot 10^{-9}$ )

forgetting factor $\lambda_{1}(0)=0.97$ (the forgetting factor is given by $\lambda_{1}(t)=\lambda_{0} \lambda_{1}(t-1)+1-\lambda_{0}$, with $\left.0<\lambda_{0}<1\right)$. For the variable forgetting factor the adaptation gain tends asymptotically towards zero. The convergence criterion has been fixed as a threshold on the trace value of the adaptation gain matrix. For details see [24].

The detection of a change of frequency is done using the variance of the measured residual force computed on a sliding window of 50 samples.

2) Protocol 2 : Adaptive operation. Real time experimental results: The measured residual force obtained in adaptive operation is presented in Fig. 8 for the direct adaptation method and in Fig. 9 for the indirect one. An adaptation gain with variable forgetting factor combined with a constant trace [29] has been used in order to be able to track automatically the changes of disturbance characteristics. The attenuation obtained with the indirect adaptive scheme is less good than in the self tuning operation and less good than the one obtained with the direct adaptive scheme.

Note: In adaptive operation, the parameters of the controller have to be re-computed at each sampling instant based on the current estimation of the disturbance model (non vanishing adaptation gain). Unfortunately, when the estimated frequency approaches the true one, the output sensitivity function will have a pair of complex zeros on the unit circle leading to a very strong attenuation of the measured effect of the disturbance. This will make the estimation of the exact frequency almost impossible. As a consequence, in adaptive operation there will be a "bias" on the estimated frequency caused by the need to have a certain level of the measured output to carry on the estimation ${ }^{8}$.

The spectral densities of the residual force (after the algorithm converges) are similar with those obtained in self-

\footnotetext{
${ }^{8}$ This phenomenon has been clearly observed both in simulation and on the real system and has also been discussed in the literature [19], [15].
}

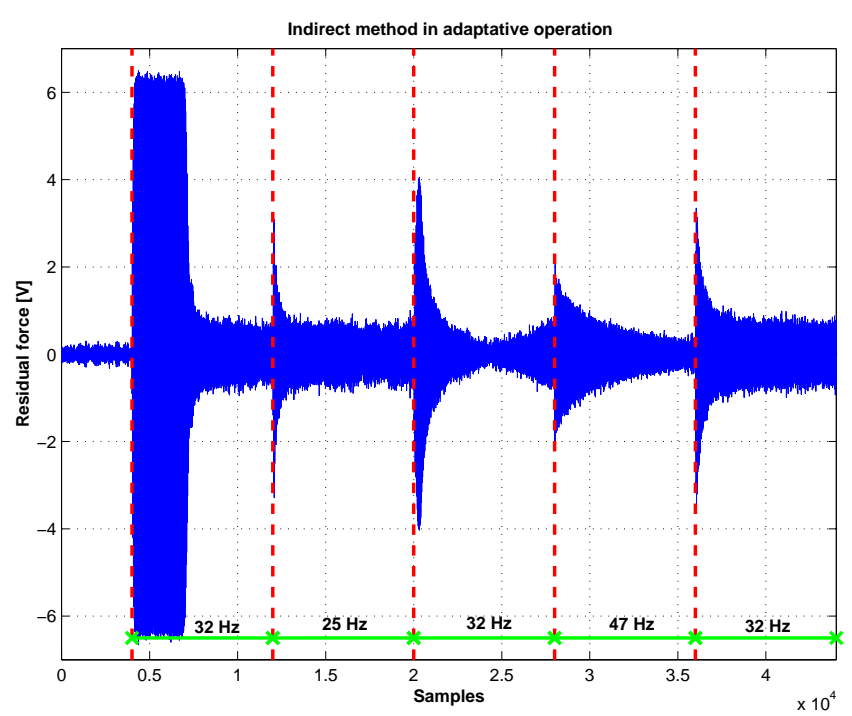

Fig. 9. Time domain results with the indirect adaptation method in the adaptive case (trace $=5 \cdot 10^{-7}$ )

tuning operation [24].

According to the real time results presented above, one can conclude that the direct adaptive control scheme gives better results than the indirect one, from the point of view of the convergence speed and performance. In addition, the direct adaptation scheme is much simpler than the indirect one in terms of number of operations.

\section{B. Linear swept frequency sinusoidal disturbances (direct adaptive control)}

Consider now that the frequency of the sinusoidal disturbance varies continuously and let's use a chirp disturbance signal (linear swept-frequency signal) between 25 and $47 \mathrm{~Hz}$. The tests have been done as follows: Start up in closed loop at $t=0$ with the central controller. Once the loop is closed, the adaptation algorithm works permanently and the controller is updated (direct approach) at each sampling. After 5 seconds a sinusoidal disturbance of $25 \mathrm{~Hz}$ (constant frequency) is applied on the shaker. From 10 to 15 seconds a chirp between 25 and $47 \mathrm{~Hz}$ is applied. After 15 seconds a $47 \mathrm{~Hz}$ (constant frequency) sinusoidal disturbance is applied and the tests are stopped after 18 seconds. The time-domain results obtained in open and in closed-loop (direct adaptive control) are presented in Fig. 10. We can remark that the performances obtained are very good.

\section{CONCLUSIONS}

It was shown in this paper that the use of the internal model principle combined with the adaptation of the internal model implemented in the controller allows a very good rejection of the unknown narrow band disturbances in active suspension systems without requiring the use of an additional transducer. Two adaptive approaches (direct and indirect adaptation) have been presented and tested comparatively on an active suspension. 

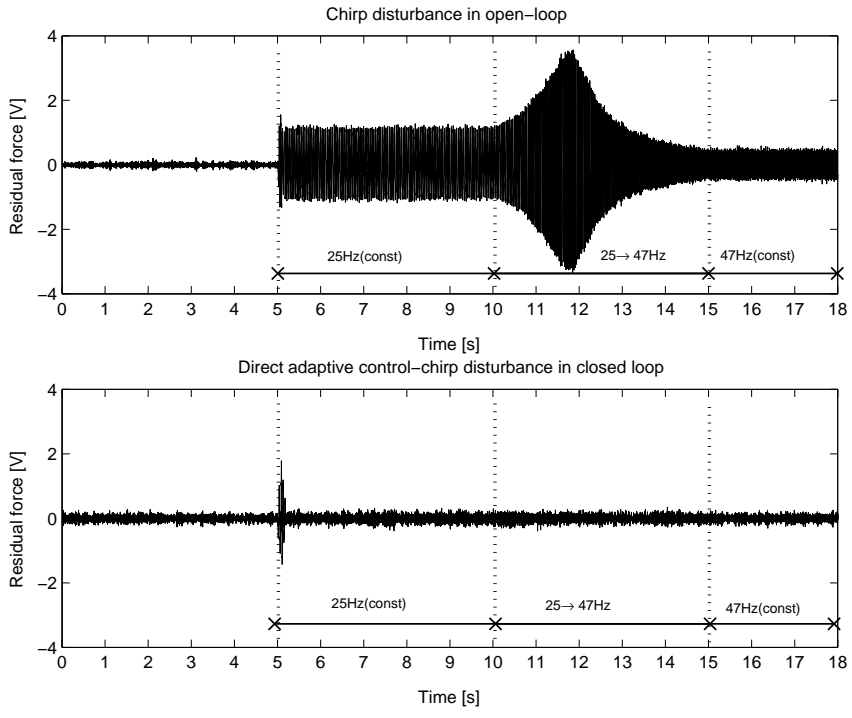

Fig. 10. Real-time results obtained with the direct adaptive method and a chirp disturbance: (a) Open loop; (b) Closed loop

The results obtained in real time lead us to conclude that the direct adaptive control scheme provides better performance than the indirect one. Furthermore, from the performances point of view, the adaptive operation is more interesting than the self-tuning one for the direct adaptive control scheme. Moreover, the direct algorithm is much simpler than the indirect one.

\section{ACKNOWLEDGMENTS}

The authors gratefully acknowledge the contribution of Prof. Ioan D. Landau at the development of the work presented in this paper.

\section{REFERENCES}

[1] S. Elliott and P. Nelson, "Active noise control," Noise / News International, pp. 75-98, June 1994.

[2] S. Elliott and T. Sutton, "Performance of feedforward and feedback systems for active control," IEEE Transactions on Speech and Audio Processing, vol. 4, no. 3, pp. 214-223, May 1996.

[3] L. Beranek and I. Ver, Noise and vibration control engineering: principles and applications. New York: Wiley, 1992.

[4] C. Fuller, S. Elliott, and P. Nelson, Active control of vibration. New York: Academic Press, 1995.

[5] B. Widrow and S. Stearns, Adaptive signal processing. Englewood Cliffs, New Jersey: Prentice-Hall, 1985.

[6] G. Feng and M. Palaniswami, "A stable adaptive implementation of the internal model principle," IEEE Trans. on Automatic Control, vol. 37, pp. $1220-1225,1992$.

[7] Z. Sun and T. Tsao, "Adaptive control with asymptotic tracking performance and its application to an electro-hydraulic servo system," Journal of Dynamic Systems Measurement and Control, vol. 122, pp. 188-195, 2000.

[8] Y. Zhang, P. Mehta, R. Bitmead, and C. Johnson, "Direct adaptive control for tonal disturbance rejection," Proceedings of the American Control Conference, Philadelphia, pp. 1480-1482, 1998.

[9] B. Francis and W. Wonham, "The internal model principle of control theory," Automatica, vol. 12, pp. 457-465, 1976.

[10] C. Johnson, "Theory of disturbance-accomodating controllers," In Control and Dynamical Systems (C. T. Leondes, Ed.), 1976, vol. 12, pp. 387-489.

[11] G. Bengtsson, "Output regulation and internal models - a frequency domain approach," Automatica, vol. 13, pp. 333-345, 1977.
[12] Y. Tsypkin, "Stochastic discrete systems with internal models," Journal of Automation and Information Sciences, vol. 29, no. 4\&5, pp. 156-161, 1997.

[13] S. Valentinotti, "Adaptive rejection of unstable disturbances : application to a fed-batch fermentation," Thèse de doctorat, École Polytechnique Fédérale de Lausanne, April 2001.

[14] F. B. Amara, P. Kabamba, and A. Ulsoy, "Adaptive sinusoidal disturbance rejection in linear discrete-time systems-part 1 and2," Journal of Dynamic Systems Measurement and Control, vol. 121, pp. 648-659, 1999.

[15] T. Gouraud, M. Gugliemi, and F. Auger, "Design of robust and frequency adaptive controllers for harmonic disturbance rejection in a single-phase power network," Proceedings of the European Control Conference, Bruxelles, 19797.

[16] G. Hillerstrom and J. Sternby, "Rejection of periodic disturbances with unknown period-a frequency domain approach," Proceedings of American Control Conference, Baltimore, pp. 1626-1631, 1994.

[17] R. Marino, G. Santosuosso, and P. Tomei, "Robust adaptive compensation of biased sinusoidal disturbances with unknown frequency," Automatica, vol. 39, pp. 1755-1761, 2003.

[18] Z. Ding, "Global stabilization and disturbance suppression of a class of nonlinear systems with uncertain internal model," Automatica, vol. 39, pp. 471-479, 2003.

[19] M. Bodson and S. Douglas, "Adaptive algorithms for the rejection of sinusosidal disturbances with unknown frequency," Automatica, vol. 33, pp. 2213-2221, 1997.

[20] I. Landau, N. M'Sirdi, and M. M'Saad, "Techniques de modélisation récursive pour l'analyse spectrale paramétrique adaptative," Revue de Traitement du Signal, vol. 3, pp. 183-204, 1986.

[21] L. Ljung, System Identification - Theory for the User, 2nd ed. Englewood Cliffs: Prentice Hall, 1999.

[22] Y. Tsypkin, "Adaptive-invariant discrete control systems," In Foundations of Adaptive Control (M. Thoma and A. Wyner, Eds.), 1991, vol. 160 of Lecture Notes in Control and Information Science, pp. 239-268, Springer Verlag.

[23] B. Anderson, "From Youla-Kucera to identification, adaptive and nonlinear control," Automatica, vol. 34, pp. 1485-1506, 1998.

[24] A. Constantinescu, "Commande robuste et adaptative d'une suspension active," Thèse de doctorat, Institut National Polytechnique de Grenoble, décembre 2001.

[25] I. Landau, A. Karimi, and A. Constantinescu, "Direct controller order reduction by identification in closed loop," Automatica, no. 37, pp. $1689-1702,2001$

[26] I. Landau, A. Constantinescu, P. Loubat, D. Rey, and A. Franco, "A methodology for the design of feedback active vibration control systems," Proceedings of the European Control Conference 2001, 2001, porto, Portugal.

[27] A.Karimi, "Design and optimization of restricted complexity controllers - benchmark," http://iawww.epfl.ch/News/EJC_Benchmark/, 2002.

[28] A. Constantinescu and I. Landau, "Direct controller order reduction by identification in closed loop applied to a benchmark problem," European Journal of Control, vol. 9, no. 1, 2003.

[29] I. Landau, R. Lozano, and M. M'Saad, Adaptive control. London: Springer, 1997.

[30] I. Landau, Commande des systèmes - Conception, Identification et Mise en oeuvre. Hermes Science, 2002, (WEB Address: http://landaubookic.lag.ensieg.inpg.fr).

[31] I. Landau, A. Constantinescu, and D. Rey, "Adaptive narrow band disturbance rejection applied to an active suspension - an internal model principle approach," Automatica, vol. 41, pp. 563-574, 2005. 